\title{
Hadronic parity violation and inelastic electron-deuteron scattering
}

\author{
C.-P. Liu, ${ }^{1, *}$ G. Prézeau, ${ }^{2, \dagger}$ and M. J. Ramsey-Musolf ${ }^{2,3,4, \dagger}$ \\ ${ }^{1}$ TRIUMF, 4004 Wesbrook Mall, Vancouver, British Columbia, Canada V6T 2A3 \\ ${ }^{2}$ Kellogg Radiation Laboratory, Caltech, Pasadena, California 91125 \\ ${ }^{3}$ Department of Physics, University of Connecticut, Storrs, Connecticut 06269 \\ ${ }^{4}$ Institute for Nuclear Theory, Box 351550, University of Washington, Seattle, Washington 98195-1550
}

(Received 10 December 2002; published 12 March 2003)

\begin{abstract}
We compute contributions to the parity-violating (PV) inelastic electron-deuteron scattering asymmetry arising from hadronic PV. While hadronic PV effects can be relatively important in PV threshold electrodisintegration, we find that they are highly suppressed at quasielastic kinematics. The interpretation of the PV quasielastic asymmetry is, thus, largely unaffected by hadronic PV.
\end{abstract}

DOI: 10.1103/PhysRevC.67.035501

PACS number(s): 24.80.+y, 25.30.Fj, 24.10.Eq, 21.30.Fe

\section{INTRODUCTION}

Parity-violating (PV) inelastic electron-nucleus scattering is an important tool in the study of hadron structure [1]. In combination with $\mathrm{PV}$ elastic electron-proton $(e-p)$ scattering, measurements of the PV quasielastic (QE) electrondeuteron $(e-d)$ asymmetry allow a separate determination of the strangeness magnetic form factor, $G_{M}^{(s)}\left(Q^{2}\right)$, and the isovector axial vector form factor, $G_{A}^{(e)(T=1)}\left(Q^{2}\right)$. Knowledge of $G_{M}^{(s)}\left(Q^{2}\right)$ provides a window on the role played by sea quarks in the electromagnetic structure of the nucleon. The axial vector form factor, in contrast, is sensitive to nucleon structure effects in higher order, electroweak radiative corrections. These corrections, which depend on the species of lepton probe (hence, the " $e$ " superscript), share features with corrections relevant to other precision electroweak measurements, such as the PV asymmetry in polarized neutron $\beta$ decay. The proper interpretation of such measurements relies on an adequate understanding of electroweak radiative corrections [2].

Recently, the SAMPLE collaboration has performed separate determinations of $G_{M}^{(s)}$ and $G_{A}^{(e)(T=1)}$ at $Q^{2}$ $=0.1(\mathrm{GeV} / c)^{2}$ using PV $e-p$ and PV QE $e-d$ scattering [3-5]. The results indicate a value for $G_{A}^{(e)(T=1)}$ consistent with zero. At tree level, one expects $G_{A}^{(e)(T=1)}\left(Q^{2}=0\right)$ $=-1.267$, while radiative corrections reduce the magnitude by roughly $40 \pm 20 \%[6,7]$. These corrections include potentially significant hadronic contributions that are responsible for the estimated theoretical uncertainty. To make the measured value of $G_{A}^{(e)(T=1)}$ close to zero would require additional effects not included in the calculation of Refs. [6,7].

One possibility, which we explore in this paper, is the contribution from the PV nucleon-nucleon $(N N)$ interaction. The latter induces small admixtures of opposite parity states into the deuteron as well as the scattered $n p$ partial waves. These parity admixtures contribute to the PV asymmetry

\footnotetext{
*Electronic address: cpliu@ triumf.ca

†Electronic address: prezeau@krl.caltech.edu

‡Electronic address: mjrm@krl.caltech.edu
}

when a $\gamma$ is exchanged between the electron and target [26]. Moreover, in contrast to the effect of $Z^{0}$ exchange, these hadronic PV effects in $\gamma$-exchange give rise to a term in the asymmetry which does not vanish at $Q^{2}=0$. For sufficiently small $Q^{2}$, this term would dominate the asymmetry. One might ask, then, whether the omission of this term in the interpretation of the SAMPLE deuterium measurement is responsible for the apparent, anomalously large radiative corrections entering $G_{A}^{(e)(T=1)}$.

Below, we show that the magnitude of this $Q^{2}$-independent hadronic PV contribution is too small to account for the observed $G_{A}^{(e)(T=1)}$ effect. Based on simple scaling arguments, the relative importance of the $Q^{2}$-independent contribution-compared to the "canonical" $Z^{0}$-exchange induced asymmetry-goes as $\sim 10^{-4} \mathrm{~m}_{N}^{2} / Q^{2}$. Thus, at the SAMPLE kinematics, $Q^{2}=0.1(\mathrm{GeV} / c)^{2}$, we expect the hadronic PV contribution to generate at most a few parts in a thousand correction to the asymmetry-far short of what would be needed to close the gap between the theoretical and experimental values for $G_{A}^{(e)(T=1)}$.

We also carry out an explicit calculation of the hadronic PV contribution and verify the expectations based on these scaling arguments. Our computation follows on the work of Refs. [8,9], in which the hadronic PV contribution to PV threshold deuteron electrodisintegration was studied, and the calculation of Ref. [10], which treated PV QE $e-d$ scattering. In the latter analysis, only parity mixing in the deuteron wave function was considered. In the present study, we also include the contributions from parity mixing in the final $e-d$ scattering states as well as from PV two-body currents. Our results are consistent with both of these earlier calculations, but give a more complete treatment of the QE case.

The remainder of the paper is organized as follows. In Sec. II, we review the formalism for PV QE scattering and hadronic PV, identify the relevant operators and matrix elements to be computed, and present the scaling arguments for the relative magnitude for the hadronic PV contribution. Section III gives a discussion of the bound and scattering state wave functions, which we determine first in the plane wave approximation and subsequently using the Argonne $V_{18}$ potential. We present the results of our calculation in Sec. IV, 
where we consider two cases: threshold electrodisintegration and QE scattering. Figs. 7, 8, 12, and 13, which show various contributions to the PV asymmetries as a function of $Q^{2}$, summarize the main results of this work. A summary discussion appears in Sec. V.

\section{PV ELECTRON SCATTERING AND HADRONIC PV}

\section{A. Basic formalism}

The PV asymmetry for inclusive $e-d$ scattering of an unpolarized target can be expressed in terms of two response functions: $W^{E M}$, the parity-conserving $(\mathrm{PC})$ electromagnetic (EM) response, and $W^{P V}$, the PV response arising from the interference of EM and PV neutral current amplitudes. One may decompose the former in terms of the longitudinal and transverse response functions

$$
\begin{gathered}
W^{E M}=\left.\sum_{f}\left[v_{L} F_{L}^{2}(q)+v_{T} F_{T}^{2}(q)\right]\right|_{\omega=E_{f}-E_{i}}, \\
F_{L}^{2}(q)=\sum_{J \geqslant 0} F_{C J}^{2}(q), \\
F_{T}^{2}(q)=\sum_{J \geqslant 1}\left[F_{E J}^{2}(q)+F_{M J}^{2}(q)\right],
\end{gathered}
$$

where $v_{L, T}$ are the standard kinematic coefficients (defined later $), q^{\mu} \equiv(\omega, \vec{q})$ is the four momentum transfered into the nuclear system ( $E_{i}$ and $E_{f}$ are its initial and final energies). The $F_{X J}(q), X=C, E, M$, are the charge, transverse electric, and transverse magnetic multipole matrix elements depending on $q=|\vec{q}|$. They are defined through multipole operators, $\hat{O}^{C}=\hat{M}, \hat{O}^{E}=\hat{T}^{e l}$, and $\hat{O}^{M}=i \hat{T}^{m a g}[1,11,12]$, as [27]

$$
\begin{aligned}
F_{X J}(q)= & \frac{1}{\sqrt{2 J_{i}+1}} \sum_{T=0,1}(-1)^{T_{f}-M_{T}}\left(\begin{array}{ccc}
T_{f} & T & T_{i} \\
-M_{T_{f}} & 0 & M_{T_{i}}
\end{array}\right) \\
& \times\left\langle J_{f}, T_{f}: \vdots \hat{O}_{J, T}^{X}(q): \vdots J_{i}, T_{i}\right\rangle
\end{aligned}
$$

where the : : denotes reduced matrix elements in both angular momentum and isospin [1]. While a collective quantum label $a$ refers to $\left(E_{a}, L_{a}, S_{a}, J_{a}, M_{J_{a}}, T_{a}, M_{T_{a}}\right)$, the sum $\Sigma_{f}$ runs over all indexes except $E_{f}$ and $M_{J_{f}}$ because they have been carried out to get Eq. (1).

For the PV response, one has

$$
\begin{aligned}
W^{P V(Z)}= & \sum_{f}\left[v_{L} W_{A V}^{L}(q)+v_{T} W_{A V}^{T}(q)\right. \\
& \left.+v_{T^{\prime}} W_{V A}^{T^{\prime}}(q)\right]\left.\right|_{\omega=E_{f}-E_{i},} \\
W_{A V}^{L}(q)= & -g_{A}^{e} \sum_{J \geqslant 0} F_{C J}(q) \widetilde{F}_{C J}(q),
\end{aligned}
$$

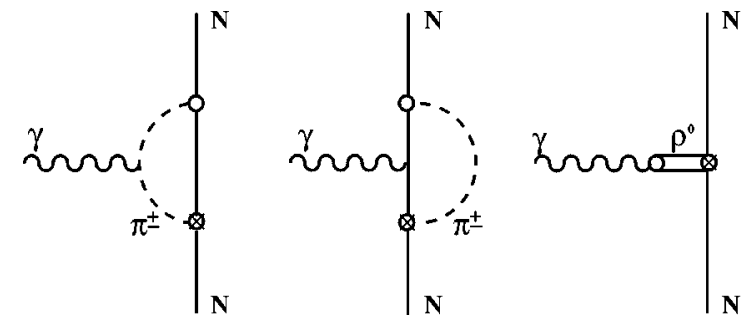

FIG. 1. Contributions due to hadronic PV in elastic $e-N$ scattering, where $\otimes$ denotes the PV meson-nucleon coupling.

$$
\begin{gathered}
W_{A V}^{T}(q)=-g_{A}^{e} \sum_{J \geqslant 1}\left[F_{E J}(q) \widetilde{F}_{E J}(q)+F_{M J}(q) \widetilde{F}_{M J}(q)\right], \\
W_{V A}^{T^{\prime}}(q)=-g_{V}^{e} \sum_{J \geqslant 1}\left[F_{E J}(q) \widetilde{F}_{M J_{5}}(q)+F_{M J}(q) \widetilde{F}_{E J_{5}}(q)\right],
\end{gathered}
$$

where the $\widetilde{F}_{X_{(5)}}$ refer to weak, neutral current multipole matrix elements and the 5 subscript indicates multipole projections of the axial vector current. The $\widetilde{F}_{X_{(5)}}$ are defined in a similar fashion as Eq. (4) —up to different coupling constants; however, for the axial form factors, it is $\hat{M}^{5}$ and $\hat{T}^{e l_{5}}$ which have additional factors of $i$ while $\hat{T}^{m^{m g_{5}}}$ is without one $[1,28]$. The kinematic coefficients, $v_{L}, v_{T}$, and $v_{T}$ are

$$
\begin{aligned}
v_{L} & =\left(Q^{2} / q^{2}\right)^{2}, \\
v_{T} & =\left(Q^{2} / q^{2}\right)^{2} / 2+\tan ^{2}(\theta / 2), \\
v_{T^{\prime}} & =\sqrt{\left(Q^{2} / q^{2}\right)^{2}+\tan ^{2}(\theta / 2)} \tan (\theta / 2),
\end{aligned}
$$

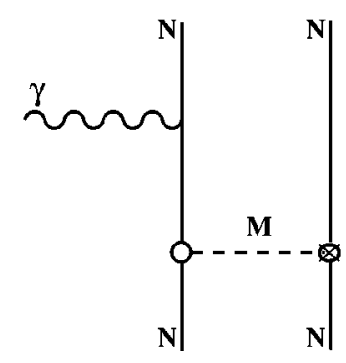

(a)

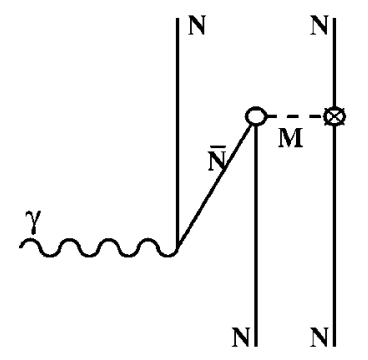

(c.1)

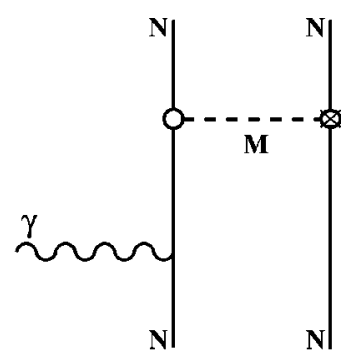

(b)

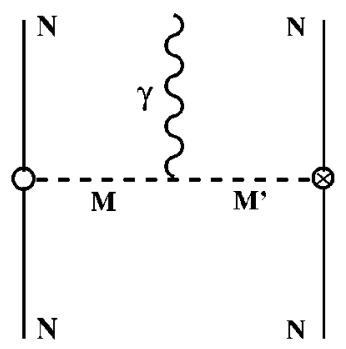

(c.2)
FIG. 2. Contributions due to two-body hadronic PV in $e-d$ scattering. Here, $M$ and $M^{\prime}$ denote the identities of mesons. 
where $Q^{2}=q^{2}-\omega^{2}, \theta$ is the scattering angle of electron.

The PV response functions $W_{A V}^{T, L}$ arise from electron axial vector $(A) \times$ hadronic vector current $(V)$ interactions, while $W_{V A}^{T^{\prime}}$ is generated by the $V(e) \times A$ (had.) interaction. At tree level in the standard model, the electron vector coupling to the $Z^{0}$ is suppressed, $g_{V}^{e}=-1+4 \sin ^{2} \theta_{W} \approx-0.1$ (the axial vector coupling is $g_{A}^{e}=1$ ).

In terms of these response functions, the PV QE asymmetry due to $Z^{0}$ exchanges is

$$
A_{L R}^{(Z)}=\frac{G_{\mu} Q^{2}}{4 \sqrt{2} \pi \alpha} \frac{W^{P V(Z)}}{W^{E M}} .
$$

For quasielastic kinematics, $\omega$ and $q$ are related, viz., $\omega$ $\approx q^{2} / 2 m_{N}$.

\section{B. Hadronic PV effects}

Hadronic PV effects in the target generate $\mathrm{O}(\alpha)$ corrections to the tree-level contributions for $W_{V A}^{T^{\prime}}$. These hadronic PV effects arise when a photon, rather than a $Z^{0}$, is exchanged between the electron and hadron. Because the vector yee coupling $Q^{e}=-1$ is an order of magnitude larger than $g_{V}^{e}$, one expects the relative importance of the hadronic PV effects-compared to the tree-level amplitude- to be of order

$$
R_{A} \sim-\frac{8 \sqrt{2} \pi \alpha}{G_{\mu} \Lambda_{\chi}^{2}} \frac{1}{1-4 \sin ^{2} \theta_{W}} \frac{g_{\pi}}{g_{A}} \approx-0.01,
$$

where $g_{\pi}=3.8 \times 10^{-8}$ sets the scale for hadronic PV interactions, $\Lambda_{\chi}=4 \pi F_{\pi} \approx 1 \mathrm{GeV}$ gives the scale of chiral symmetry breaking, and $g_{A}=1.267 \pm 0.004$. In the case of elastic $e-N$ scattering, hadronic $\mathrm{PV}$ arises via diagrams of the type in Fig. 1. These corrections induce a PV $\gamma N N$ coupling, or anapole moment. The latter has been used in the one-body estimate of $R_{A}$ given in Refs. [6,7]. Those analyses indicate hadronic PV-induced anapole corrections of $-6 \pm 20 \%$.

Two-body hadronic PV contributions arise from the diagrams in Fig. 2. Figs. 2(a) and (b) indicate parity mixing in the initial and final state wave functions, while Fig. 2(c) indicates the PV two-body EM current contribution. Each contributes to an effective axial vector EM transition amplitude, whose effects appear as corrections to the $\widetilde{F}_{X J_{5}}(q)$ multipole matrix elements.

In computing the parity-mixing matrix elements, we use the model PV Hamiltonian given in Ref. [13]:

$$
\begin{aligned}
H_{P V}(\vec{r})= & \frac{g_{\pi N N} h_{\pi}^{1}}{4 \sqrt{2} m_{N}}\left(i \vec{\tau}_{1} \times \vec{\tau}_{2}\right)_{z}\left(\vec{\sigma}_{1}+\vec{\sigma}_{2}\right) \cdot \vec{u}_{\pi}(\vec{r}) \\
& -\frac{g_{\rho N N}}{2 m_{N}}\left(h_{\rho}^{0} \vec{\tau}_{1} \cdot \vec{\tau}_{2}+\frac{h_{\rho}^{1}}{2}\left(\vec{\tau}_{1}+\vec{\tau}_{2}\right)_{z}+\frac{h_{\rho}^{2}}{2 \sqrt{6}}\left(3 \tau_{1 z} \tau_{2 z}-\vec{\tau}_{1} \cdot \vec{\tau}_{2}\right)\right)\left[\left(1+\mu_{v}\right) i \vec{\sigma}_{1} \times \vec{\sigma}_{2} \cdot \vec{u}_{\rho}(\vec{r})+\left(\vec{\sigma}_{1}-\vec{\sigma}_{2}\right) \cdot \vec{v}_{\rho}(\vec{r})\right] \\
& \left.-\frac{g_{\omega N N}}{2 m_{N}}\left(h_{\omega}^{0}+\frac{h_{\omega}^{1}}{2}\left(\vec{\tau}_{1}+\vec{\tau}_{2}\right)_{z}\right)\left[\left(1+\mu_{s}\right) i \vec{\sigma}_{1} \times \vec{\sigma}_{2}\right] \cdot \vec{u}_{\omega}(\vec{r})+\left(\vec{\sigma}_{1}-\vec{\sigma}_{2}\right) \cdot \vec{v}_{\omega}(\vec{r})\right] \\
& -\frac{1}{4 m_{N}}\left(\vec{\tau}_{1}-\vec{\tau}_{2}\right)_{z}\left(\vec{\sigma}_{1}+\vec{\sigma}_{2}\right) \cdot\left[g_{\omega N N} h_{\omega}^{1} \vec{v}_{\omega}(\vec{r})-g_{\rho N N} h_{\rho}^{1} \vec{v}_{\rho}(\vec{r})\right]-\frac{g_{\rho N N}}{4 m_{N}} h_{\rho}^{1^{\prime}}\left(i \vec{\tau}_{1} \times \vec{\tau}_{2}\right)_{z}\left(\vec{\sigma}_{1}+\vec{\sigma}_{2}\right) \cdot \vec{u}_{\rho}(\vec{r})
\end{aligned}
$$

where the $h_{M}^{(X)}$ and $g_{M N N}$ are the weak, PV and strong, PC meson-nucleon couplings, respectively, the strong scalar and vector anamalous magnetic moments have the values: $\mu_{s}=$ -0.12 and $\mu_{v}=3.70$, and $\vec{u}_{\alpha}(\vec{r})=\left[\vec{p}_{1}-\vec{p}_{2}, e^{-m_{\alpha} r} / r\right]$, $\vec{v}_{\alpha}(\vec{r})=\left\{\vec{p}_{1}-\vec{p}_{2}, e^{-m_{\alpha} r} / r\right\}$. Values for the $h_{M}^{(X)}$ have been predicted theoretically using a variety of approaches. For purposes of our calculation, we will adopt the so-called DDH "best values" and "reasonable ranges" of Ref. [13]. The latter are consistent with constraints obtained from a variety of hadronic and nuclear PV experiments, as discussed in Refs. $[14,15]$.

Current conservation requires that one include contributions from PV two-body currents to the PV multipole matrix elements. These currents have been derived in Ref. [16,17] from the diagrams in Fig. 2(c). Complete expressions for the coordinate space current operators could be found in Ref.
[17]. To illustrate the structure of these operators, however, we give the complete two-body current operator associated with the $\pi^{ \pm}$-exchange component of $H_{P V}$ :

$$
\begin{aligned}
J_{\mu}\left(\vec{y}, \vec{x}_{1}, \vec{x}_{2}\right)^{P V, \pi} & \frac{-e g_{\pi N N} h_{\pi}^{1}}{8 \sqrt{2} \pi m_{N}}\left(\vec{\tau}_{1} \cdot \vec{\tau}_{2}-\tau_{1 z} \tau_{2 z}\right)\left[\vec{\sigma}_{1} \delta^{(3)}\left(\vec{y}-\vec{x}_{1}\right)\right. \\
& +\vec{\sigma}_{2} \delta^{(3)}\left(\vec{y}-\vec{x}_{2}\right)-\frac{1}{2}\left(\vec{\sigma}_{1} \cdot \vec{\nabla}_{1}-\vec{\sigma}_{2} \cdot \vec{\nabla}_{2}\right)\left(\vec{x}+\frac{1}{2} \frac{x}{m_{\pi}} \vec{\nabla}_{y}\right) \\
& \times\left[\delta^{(3)}\left(\vec{y}-\vec{x}_{1}\right)+\delta^{(3)}\left(\vec{y}-\vec{x}_{2}\right)\right]\left[\frac{e^{-m_{\pi^{x}}}}{x}, \quad \mu=1,2,3\right. \\
\approx & O(v / c)^{2}, \quad \mu=0
\end{aligned}
$$


where $\vec{x}_{i}$ denotes the position of the $i$ th nucleon and $x=|\vec{x}|$ $=\left|\vec{x}_{1}-\vec{x}_{2}\right|$. Inclusion of these PV two-body currents guarantees that the continuity equation, $\vec{\nabla} \cdot \vec{J}=i[\hat{H}, \rho]$, is satisfied at the operator level for the PV NN interaction.

Current conservation may also be implemented in writing down the various multipole operators. Since nuclear model calculations based on realistic potentials generally break current conservation, it is useful to implement the latter via the form of the multipole operator. A well-known example is Siegert's theorem [18], which allows one to rewrite the $J$ $=1$ transverse electric multipole operator, $\hat{T}_{J=1}^{e l}$, in terms of the electric dipole operator (the $J=1$ charge multiple) in the long wavelength limit. An extended version of this theorem [19] allows one to implement the constraints of current conservation for transverse electric operators of arbitrary $J$ and momentum transfer. In general, one has [20]

$$
\hat{T}_{J}^{e l}(q)=\hat{S}_{J}^{e l}(q)+\hat{R}_{J}^{e l}(q) .
$$

For $J=1$, one has

$$
\begin{gathered}
\hat{S}_{1 M_{J}}^{e l}(q)=\frac{\sqrt{2}}{3} \int d^{3} x x\left[Y_{1 M_{J}}\left(\Omega_{x}\right) \rho(\vec{x}), \hat{H}\right], \\
\hat{R}_{1 M_{J}}^{e l}(q)=-\frac{q^{2}}{9} \int d^{3} x x \vec{Y}_{111}^{M_{J}}\left(\Omega_{x}\right) \cdot \vec{x} \times \vec{j}(\vec{x}),
\end{gathered}
$$

where $\vec{Y}_{J L 1}^{M_{J}}$ is the vector spherical harmonic.

Note that in the long wavelength limit, $\hat{S}_{1}^{e l}(q)$ gives the leading contribution to $F_{E 1}, \widetilde{F}_{E 1}$, and $\widetilde{F}_{E 1_{5}}$. For elastic electron scattering, hermiticity and time-reversal invariance require that $F_{E 1}$ and $\widetilde{F}_{E 1}$ must vanish. Moreover, contributions from $\hat{S}_{1}^{e l_{5}}(q)$ also vanish for elastic scattering, since the commutator in Eq. (17) leads to a factor of $\omega=E_{f}-E_{i}=0$. A non-vanishing contribution arises from $\hat{R}_{1}^{e l_{5}}(q)$, whose matrix element constitutes the nuclear anapole moment contribution. The latter is proportional to $Q^{2}$, which cancels the $1 / Q^{2}$ from the photon propagator to produce a $Q^{2}$-independent scattering amplitude at lowest order. This contribution is kinematically indistinguishable from the $Z^{0}$-exchange contribution to $\widetilde{F}_{E 1_{5}}$ and, thus, represents a simple multiplicative correction to the tree-level axial vector response.

For the inelastic transition of interest here, matrix elements of $\hat{S}_{1}^{e l_{5}}(q)$ do not vanish, nor do they contain a power of $Q^{2}$ to cancel the $1 / Q^{2}$ from the photon propagator $\left(q^{2}\right.$ $\approx Q^{2}$ at low energies). The scattering amplitude associated with this operator goes as $1 / Q^{2}$ at low $Q^{2}$. When multiplied by the factor of $Q^{2}$ in Eq. (12), this term thus generates a $Q^{2}$-independent, nonvanishing contribution to $A_{L R}$ at low $Q^{2}$, in contrast to the $Z^{0}$-exchange asymmetry that vanishes at $Q^{2}=0$. For sufficiently low $Q^{2}$, the $Q^{2}$-independent hadronic PV EM contribution will dominate the asymmetry. As we show below, such a kinematic region may be reached in principle with threshold PV electrodisintegration. For PV QE scattering at the kinematics of Ref. [5], however, the effects of hadronic PV appear to be negligible.

At the kinematics of QE electron scattering, a tower of final state partial waves contribute to the amplitude, and one must include the effects of $J>1$ multipole matrix elements (both transverse electric and transverse magnetic). This situation contrasts with threshold disintegration, where only the lowest $J$ partial waves may be reached. As we show in Sec. IV, the multipole contributions to the PV QE asymmetry (due to hadronic PV) saturate for $J \sim 7$. All multipole matrix elements having $J>1$ carry factors of $Q^{2}$, so that they do not contribute to the $Q^{2}$-independent term in the PV asymmetry. Nevertheless, the sum of their effects represents a tiny correction to the $Z^{0}$-exchange asymmetry.

It is useful to illustrate how PV NN effects contribute to the various multipole matrix elements entering the axial response, $W_{V A}^{T^{\prime}}$. Consider, for example, a transition from the deuteron ground state to the ${ }^{1} S_{0}$ continuum state. In the absence of the tensor force component of the strong $N N$ interaction, the deuteron ground state is pure ${ }^{3} S_{1}$. The PV $N N$ interaction will mix $P$ states into these $S$ waves. In ordinary perturbation theory, one has

$$
\begin{aligned}
& \left.\left|{ }^{3} S_{1}\right\rangle \rightarrow\left|{ }^{3} S_{1}\right\rangle+\widetilde{{ }^{3} S_{1}}\right\rangle, \\
& \left|{ }^{1} S_{0}\right\rangle \rightarrow\left|{ }^{1} S_{0}\right\rangle+\left|\widetilde{{ }^{1} S_{0}}\right\rangle,
\end{aligned}
$$

where the parity mixtures (denoted with a tilde " ") are

$$
\begin{aligned}
& \left|\widetilde{{ }^{3} S_{1}}\right\rangle=\sum_{k=1,3}\left|{ }^{k} P_{1}\right\rangle \frac{\left\langle{ }^{k} P_{1}\left|H_{P V}\right|{ }^{1} S_{0}\right\rangle}{E_{0}-E_{k}}, \\
& \left|\widetilde{{ }^{T} S_{0}}\right\rangle=\left|{ }^{3} P_{0}\right\rangle \frac{\left\langle{ }^{3} P_{0}\left|H_{P V}\right|{ }^{1} S_{0}\right\rangle}{E_{0}^{\prime}-E_{1}^{\prime}}
\end{aligned}
$$

For this $J_{i}=1$ to $J_{f}=0$ transition, only $J=1$ multipole operators contribute. For the PC $\gamma$-exchange contribution, one has only the magnetic dipole transition between the unmixed ${ }^{3} S_{1}$ and ${ }^{1} S_{0}$ initial and final state components, resulting in a nonzero $F_{M 1}$ form factor. For the PV $Z^{0}$-exchange amplitude, only the operator $\hat{T}_{1}^{e l_{5}}(q)$ contributes, connecting the unmixed ${ }^{3} S_{1}$ and ${ }^{1} S_{0}$ components and leading to a nonzero $\widetilde{F}_{E 1_{5}}$. The PV $N N$ interaction also contributes to the latter in three ways: (a) a nonvanishing matrix element of $\hat{T}_{1}^{e l}(q)$ between the initial state $\left|{ }^{3} S_{1}\right\rangle$ and the final state $\left|{ }^{3} P_{0}\right\rangle$ parity admixture, (b) nonvanishing matrix elements of $\hat{T}_{1}^{e l}(q)$ between the $\left|{ }^{1,3} P_{1}\right\rangle$ mixture in the initial state and the final state $\left|{ }^{1} S_{0}\right\rangle$, (c) matrix elements of the PV two-body current operator $\hat{T}_{1}^{e l_{5}}(q)$ between the $\left|{ }^{3} S_{1}\right\rangle$ and $\left|{ }^{1} S_{0}\right\rangle$ components. All other contributions are higher-order in the weak interaction and can be neglected. The analysis is similar when the $D$-state components of the deuteron and scattering state induced by the tensor force are included, as is the analysis for transitions to higher partial waves. 
Because the hadronic PV contributes to the asymmetry by inducing axial photonic couplings, to incorporate these in the expression of Eq. (12), one only has to modify axial form factors $\widetilde{F}_{X_{5}}$ by

$$
\widetilde{F}_{X J_{5}} \rightarrow \widetilde{F}_{X J_{5}}+\beta \widetilde{F}_{X J_{5}}^{(\gamma)}
$$

where

$$
\beta=-\frac{8 \sqrt{2} \pi \alpha}{G_{\mu} Q^{2}} \frac{Q^{e}}{g_{V}^{e}} .
$$

The EM axial form factors may be decomposed as

$$
\begin{aligned}
\widetilde{F}_{X J_{5}}^{(\gamma)}= & \frac{1}{\sqrt{2 J_{i}+1}} \sum_{T=0,1}(-1)^{T_{f}-M_{T}}\left(\begin{array}{ccc}
T_{f} & T & T_{i} \\
-M_{T f} & 0 & M_{T i}
\end{array}\right) \\
& \times\left\{\left\langle J_{f}, T_{f}: \vdots \hat{O}_{J, T}^{X}(q) \vdots: \widetilde{J}_{i}, T_{i}\right\rangle\right. \\
& +\left\langle\widetilde{J_{f}, T_{f}} \vdots \vdots \hat{O}_{J, T}^{X}(q) \vdots \vdots J_{i}, T_{i}\right\rangle \\
& \left.+\left\langle J_{f}, T_{f}: \vdots \hat{O}_{J, T}^{X}(q) \vdots \vdots J_{i}, T_{i}\right\rangle\right\},
\end{aligned}
$$

where $\left|\widetilde{J_{i}, T_{i}}\right\rangle,\left\langle\widetilde{J_{f}, T_{f}}\right|$, and $\hat{O}_{J, T}^{X_{5}}(q)$ (the two-body PV EM operators) represent the effects caused by hadronic PV. One may then express the asymmetry due to hadronic PV (through the radiative corrections) as

$$
A_{L R}^{(\gamma)}=2 \frac{W^{P V(\gamma)}}{W^{E M}}
$$

where

$$
\begin{aligned}
W^{P V(\gamma)}= & v_{T^{\prime}} \sum_{f} \sum_{J \geqslant 1}\left[F_{E J}(q) \widetilde{F}_{M J_{5}}^{(\gamma)}(q)\right. \\
& \left.+F_{M J}(q) \widetilde{F}_{E J_{5}}^{(\gamma)}(q)\right]\left.\right|_{\omega=E_{f}-E_{i}}
\end{aligned}
$$

A simple scaling argument allows us to estimate the relative impact of the two-body hadronic PV contribution. For backward-angle scattering as studied in the SAMPLE experiments, $v_{T^{\prime}} \approx v_{T} \gg v_{L}$, so the ratio of asymmetry due to hadronic PV, Eq. (26), and $Z^{0}$, Eq. (12) is

$$
\frac{A_{L R}^{(\gamma)}}{A_{L R}^{(Z)}} \approx \frac{8 \sqrt{2} \pi \alpha}{G_{\mu} Q^{2}} \frac{W^{P V(\gamma)}}{W^{P V(Z)}} \approx \frac{8 \sqrt{2} \pi \alpha}{G_{\mu} Q^{2}} \frac{\left\langle\vec{j}_{P V}^{\gamma}\right\rangle}{\left\langle\vec{j}^{Z}\right\rangle},
$$

while $\left\langle\vec{j}^{Z}\right\rangle$ at backward angles is dominated by the magnetic NC component and scales as $\langle\vec{\sigma}\rangle,\left\langle\vec{j}_{P V}^{\gamma}\right\rangle$ scales as $\langle\vec{\sigma}\rangle$ times an additional factor introduced by hadronic PV. Using Eq. (15) for a guidance, this factor is roughly

$$
\frac{g_{\pi N N} h_{\pi}^{1}}{8 \sqrt{2} \pi}\left\langle\frac{e^{-m_{\pi} x}}{m_{N} x}\right\rangle
$$

With $g_{\pi N N} \cong 13.45$, and $h_{\pi} \sim 4.5 \times 10^{-7}$, the scaling rule is (a)

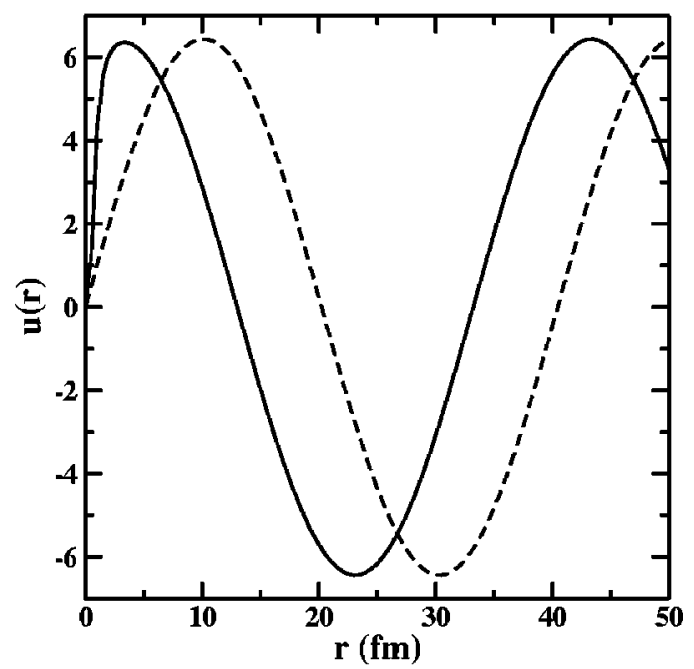

(b)

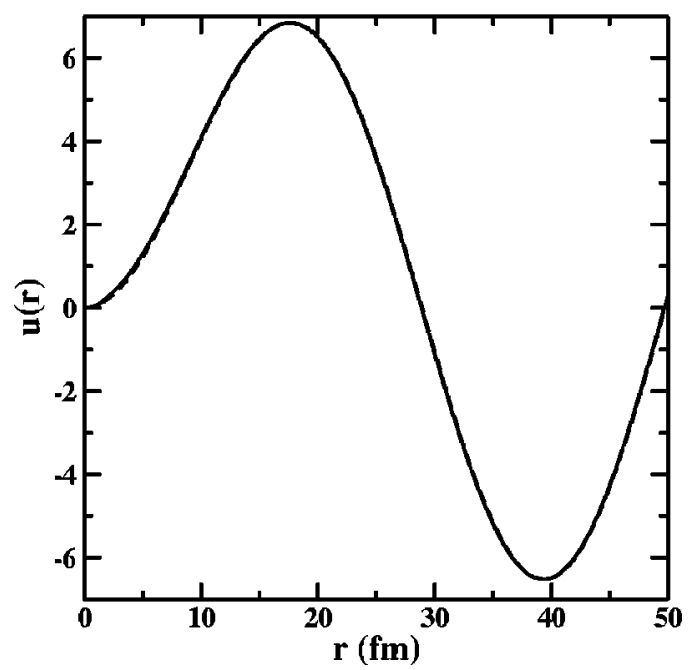

FIG. 3. Comparison of scattering state wave functions: (a) ${ }^{1} S_{0}$ channel and (b) ${ }^{3} P_{0}$ channel, where dashed lines give the plane wave solutions and solid lines give results of the potential model calculations using $\mathrm{AV}_{18}$. The relative energy of $n p$ is $1 \mathrm{MeV}$.

$$
\frac{A_{L R}^{(\gamma)}}{A_{L R}} \sim \frac{m_{N}^{2}}{Q^{2}}\left\langle\frac{e^{-m_{\pi} x}}{m_{N} x}\right\rangle \times 10^{-3}
$$

where we have also included the NC magnetic form factor $\widetilde{G}_{M}\left(Q^{2}\right)$ in the denominator. For small $Q^{2}$, one has $\widetilde{G}_{M}$ $\approx \mu_{V}=4.70$. Taking $x \sim 1 \mathrm{fm},\left\langle e^{-m_{\pi} x} /\left(m_{N} x\right)\right\rangle \sim 0.1$, therefore at $Q^{2} \sim 0.1(\mathrm{GeV} / c)^{2}$, we have $A_{L R}^{(\gamma)} / A_{L R}^{(Z)}$ in the order of a few $0.1 \%$.

\section{TWO-BODY WAVE FUNCTIONS}

In order to compute PV matrix elements in Eq. (25), we need two-body wave functions. The latter are solutions of the Schrödinger equation 
(a)

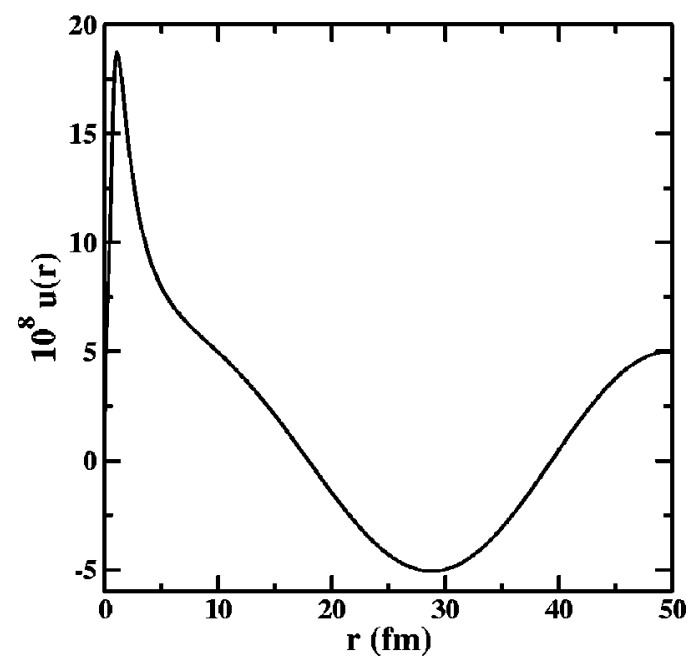

(b)

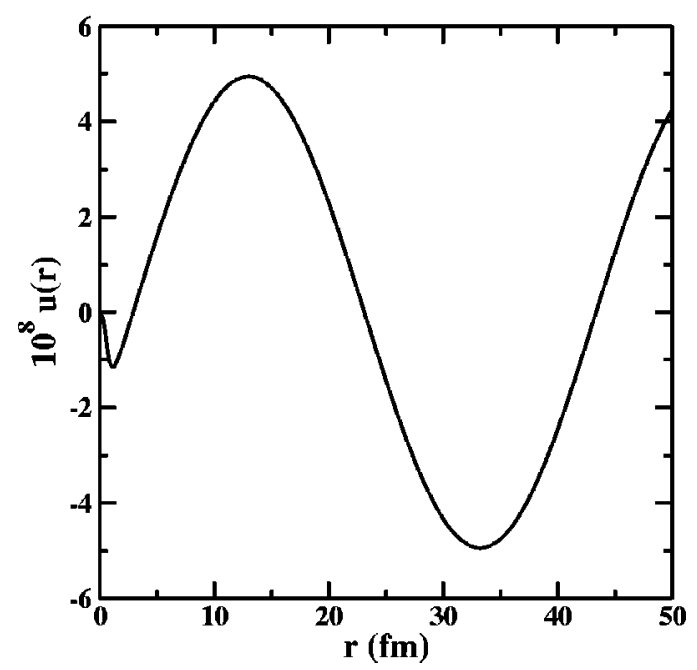

FIG. 4. PV admixtures for the scattering states in Fig. 3: (a) ${ }^{3} P_{0}$ admixture in ${ }^{1} S_{0}$ and (b) ${ }^{1} S_{0}$ admixture in ${ }^{3} P_{0}$, obtained by solving the inhomogeneous differential equations.

$$
\left(H_{0}+H_{P V}\right)|\psi+\widetilde{\psi}\rangle=E|\psi+\widetilde{\psi}\rangle .
$$

Since $H_{P V}$ is much smaller than $H_{0}$, first-order perturbation should work well in this process. That is, Eq. (31) can be solved in two steps: first, the PC part $|\psi\rangle$ is determined by solving

$$
\left(E-H_{0}\right)|\psi\rangle=0,
$$

and second, the PV admixture $|\widetilde{\psi}\rangle$ is determined from

$$
\left(E-H_{0}\right)|\widetilde{\psi}\rangle=H_{P V}|\psi\rangle,
$$

with $|\psi\rangle$ obtained in the first step. In what follows, we explore two different approaches, one using the plane wave approximation-which ignores the final state strong interaction (FSI)-and one using a potential model calculation, which includes the FSI. (a)

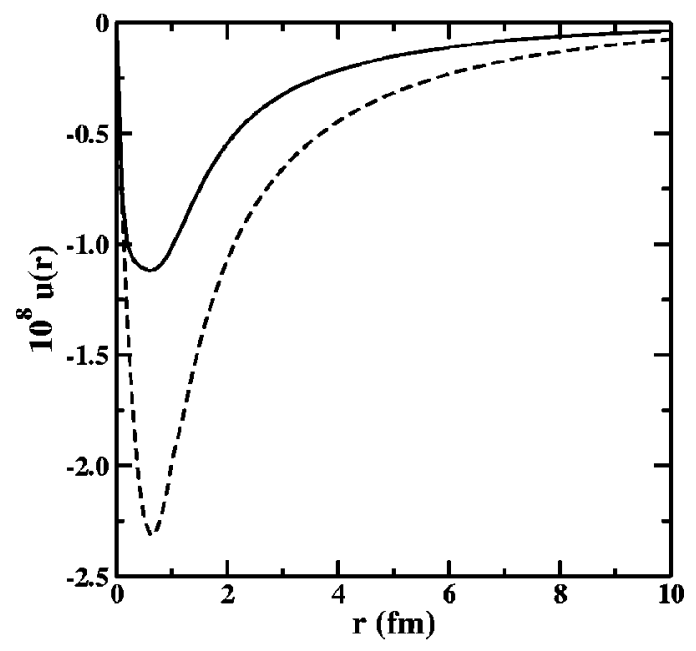

(b)

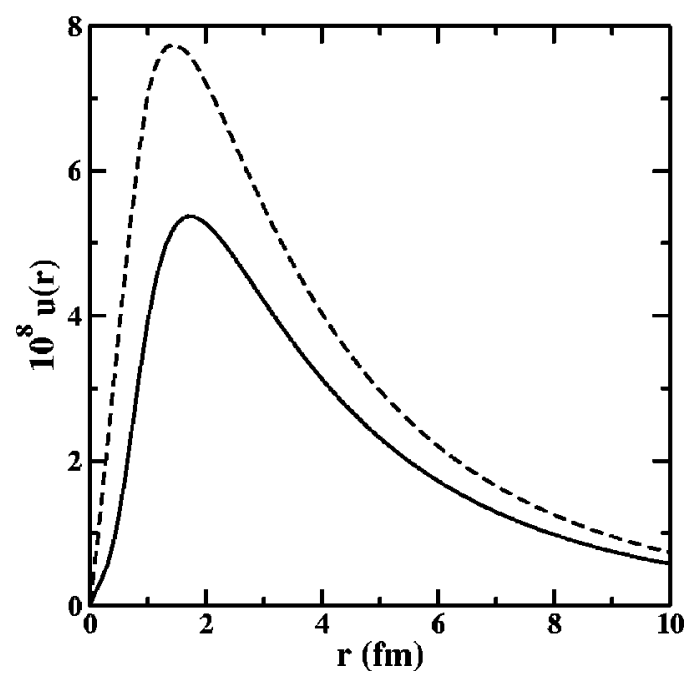

FIG. 5. PV admixtures of deuteron: (a) ${ }^{1} P_{1}$ admixture and (b) ${ }^{3} P_{1}$ admixture, dashed lines are results using the plane wave Green's function and solid lines are calculations using $\mathrm{AV}_{18}$.

\section{A. Plane wave approximation}

Although the plane wave approximation is naive and simple, we employ it as a toy-model calculation to achieve some initial insights. In addition, the computation of Ref. [10] employed a plane wave Green's function to compute the PV admixture in the deuteron, though unmixed deuteron wave function was obtained using the Bonn potential. By comparing the plane wave computation with the potential model solution (see below), we hope to obtain a sense of the errors introduced by the plane wave approximation.

In this approach, all the radial components of scattering partial waves are spherical Bessel function, $j_{L}(p r)$, where $L$ is the relative orbital angular momentum and $p$ is the relative momentum.

The parity admixture, to first order in the perturbation expansion, is expressed as 
(a)

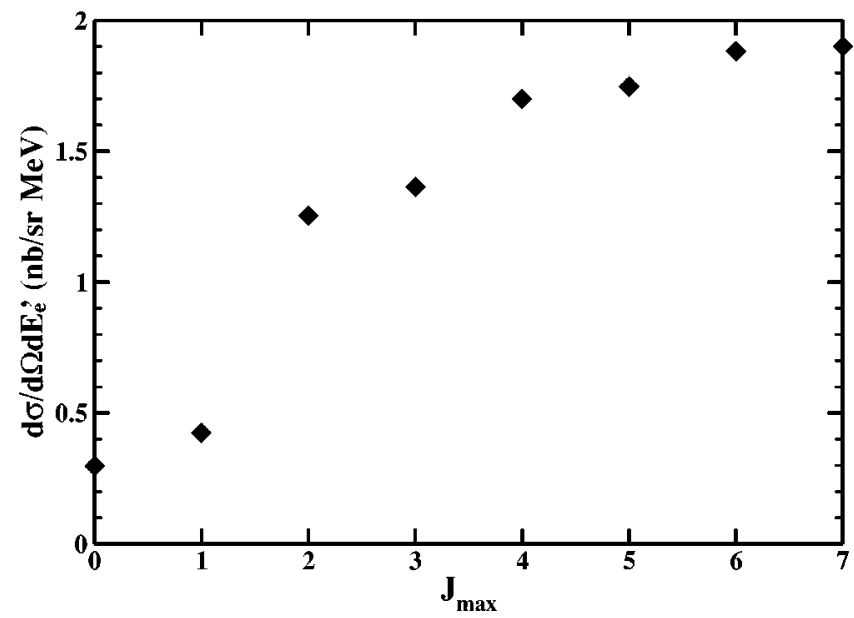

(b)

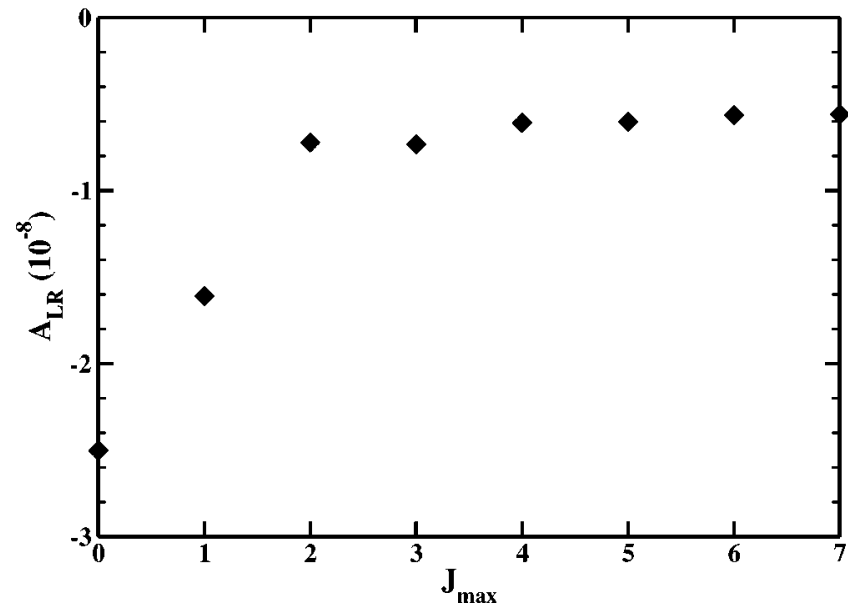

FIG. 6. The saturation behavior of (a) total cross section and (b) asymmetry as functions of $J_{\max }$, the maximum total angular momentum for final states being included.

$$
|\widetilde{\psi}\rangle=\sum_{\phi}|\phi\rangle \frac{1}{E_{\psi}-E_{\phi}}\left\langle\phi\left|H_{P V}\right| \psi\right\rangle,
$$

where $|\phi\rangle$ forms a complete eigenbasis. This could be computed if one knows Green's function

$$
G(\vec{x}, \vec{y})=\sum_{\phi} \frac{\langle\vec{x} \mid \phi\rangle\langle\phi \mid \vec{y}\rangle}{E_{\psi}-E_{\phi}}
$$

In the plane wave basis, closed-form Green's functions exist for both calculations of deuteron and final state mixing.

For the deuteron mixing, the transition involves a bound state (binding energy $E_{B}<0$ ) to continuum state transition, therefore, $E_{B}-E_{\phi}<0$. Green's function is

$$
\begin{aligned}
G^{(\mathcal{D})}(\vec{x}, \vec{y})= & \sum_{L, S, J, M_{J}, T, M_{T}} \delta_{L S T}\left(-\frac{2}{\pi} \gamma m_{N}\right) i_{L}\left(\gamma r_{<}\right) k_{L}\left(\gamma r_{>}\right) \\
& \times \mathcal{Y}_{J L S}^{\dagger M_{J}}\left(\Omega_{x}\right) \mathcal{Y}_{J L S}^{M_{J}}\left(\Omega_{y}\right) \chi_{T}^{\dagger M_{T}} \chi_{T}^{M_{T}},
\end{aligned}
$$

(a)

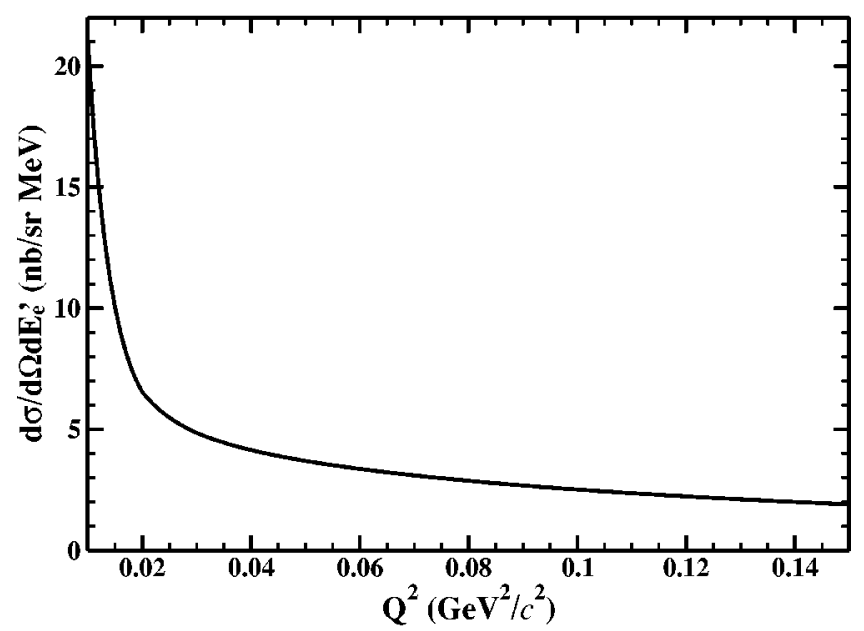

(b)

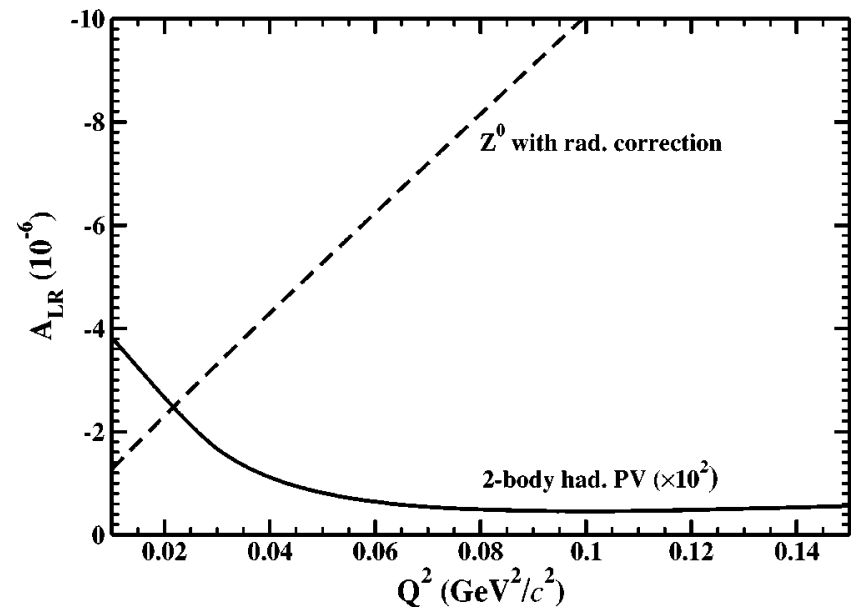

FIG. 7. The (a) total cross section and (b) PV asymmetry versus $Q^{2}$, where the kinematics are constrained to satisfy $q^{2}=2 m_{N} \omega$ and $\theta=180^{\circ}$. Note in (b), the hadronic PV contribution is multiplied by 100 ; it does not actually cross the $Z^{0}$ term in this $Q^{2}$ range.

where $\gamma=\sqrt{m_{N}\left|E_{B}\right|}, i_{L}(\gamma r)$ and $k_{L}(\gamma r)$ are the modified spherical Bessel functions of the first and third kind, $r_{<}\left(r_{>}\right)$ refers to the smaller (larger) radial coordinate of $x$ and $y, \mathcal{Y}$ and $\chi$ denote the spin-angular and isospin wave functions. The factor $\delta_{L S T}$, which is 1 if $L+S+T$ is an odd number and 0 otherwise, enforces the generalized Pauli principle.

For the final state mixing, because of the pole at $E_{\psi}$ $=E_{\phi}$, we must add a small imaginary number $\pm i \epsilon$ to the energy denominator, as in the scattering problem. In this way, we obtain a retarded (advanced) Green's function corresponding to the $-i \epsilon(+i \epsilon)$ prescription. However, only the real part of this Green's function gives a non-vanishing response function. The real part is equivalent to the average of retarded and advanced ones

$$
\begin{aligned}
\bar{G}^{(\mathcal{F})}(\vec{x}, \vec{y})= & \sum_{L, S, J, M_{J}, T, M_{T}} \delta_{L S T}\left(m_{N} p_{\mathcal{F}}\right) j_{L}\left(p_{\mathcal{F}} r_{<}\right) n_{L}\left(p_{\mathcal{F}} r_{>}\right) \\
& \times \mathcal{Y}_{J L S}^{\dagger M_{J}}\left(\Omega_{x}\right) \mathcal{Y}_{J L S}^{M_{J}}\left(\Omega_{y}\right) \chi_{T}^{\dagger M_{T}} \chi_{T}^{M_{T}}
\end{aligned}
$$




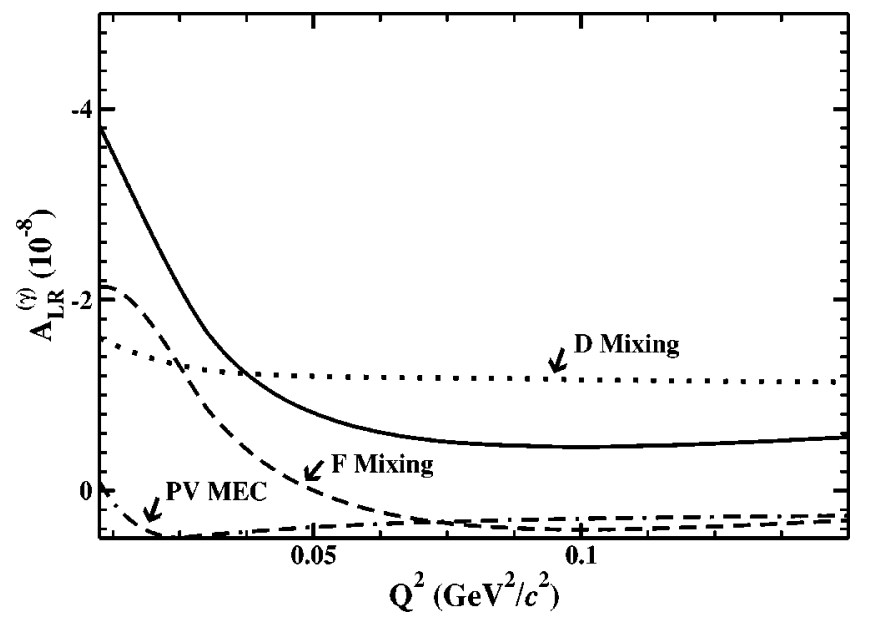

FIG. 8. The breakdown of various hadronic PV contributions to the asymmetry at QE kinematics, where $D, F$, and MEC refer to contributions from deuteron mixing, final state mixing, and PV meson exchange currents, respectively, and the solid line gives the total.

where $p_{\mathcal{F}}$ is the relative momentum of the final state and $n_{L}\left(p_{\mathcal{F}} r\right)$ is the spherical Neumann wave functions.

Illustrative results for the plane wave calculation are given in Figs. 3 and 5. We note that, in comparison with the complete, coupled channel potential model computation (see below), use of the plane wave Green's function overestimates the degree of parity mixing in the deuteron ground state. For parity-mixing in the scattering states, we also find a mismatch between the two approaches, though no systematic pattern emerges as to the magnitude or sign of the difference. The problem may be particularly severe for the ${ }^{3} S_{1}$ and ${ }^{3} D_{1}$ scattering states which, in the plane wave approach, are not automatically orthogonal to the deuteron wave function $|\mathcal{D}\rangle$. Although one might attempt to solve this problem by implementing orthogonality by hand, viz.,

$$
\left|{ }^{3} S_{1},{ }^{3} D_{1}\right\rangle_{\perp}=\left|{ }^{3} S_{1},{ }^{3} D_{1}\right\rangle-|\mathcal{D}\rangle\left\langle\left.\mathcal{D}\right|^{3} S_{1},{ }^{3} D_{1}\right\rangle,
$$

it is questionable whether this ad hoc solution is rigorously correct. For these reasons, then, we rely only on the coupled channel potential model computation to determine the nuclear PV contribution to the inelastic asymmetry.

\section{B. Potential model calculation}

Although the $N N$ potential determined directly from solving QCD is not available, a variety of modern phenomenological potentials successfully fit $N N$ scattering data (below $350 \mathrm{MeV}$ or so) as well as deuteron properties with reasonable $\chi^{2}$ values. Here, we use the Argonne $V_{18}$ potential $\left(\mathrm{AV}_{18}\right)[21]$

The PC scattering wave function,

$$
\left\langle\vec{r} \mid E, L, S, J, M_{J}, T, M_{T}\right\rangle=\sqrt{\frac{2 m_{N} k}{\pi}} \frac{u_{J L S}(r)}{r} \mathcal{Y}_{J L S}^{M_{J}} \chi_{T}^{M_{T}},
$$

(a)

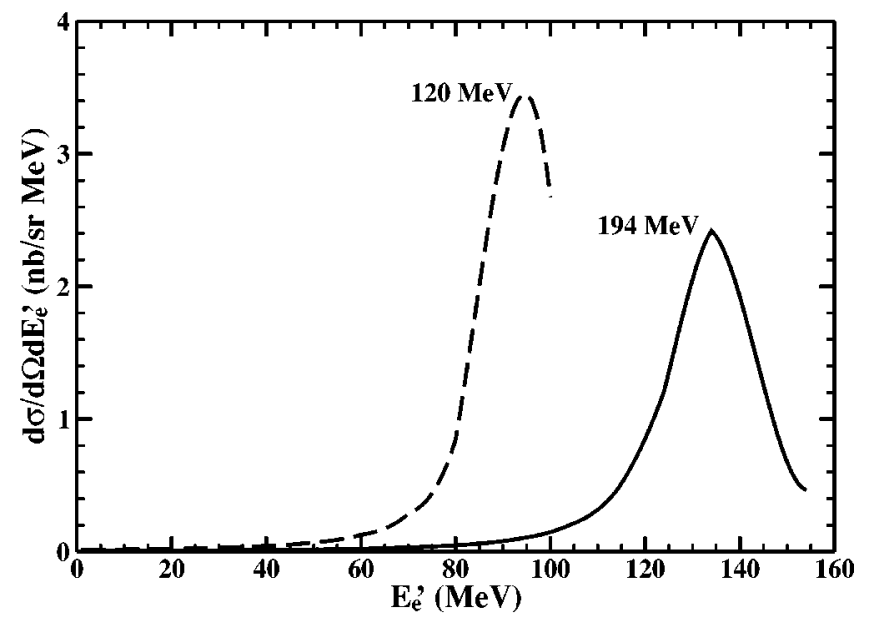

(b)

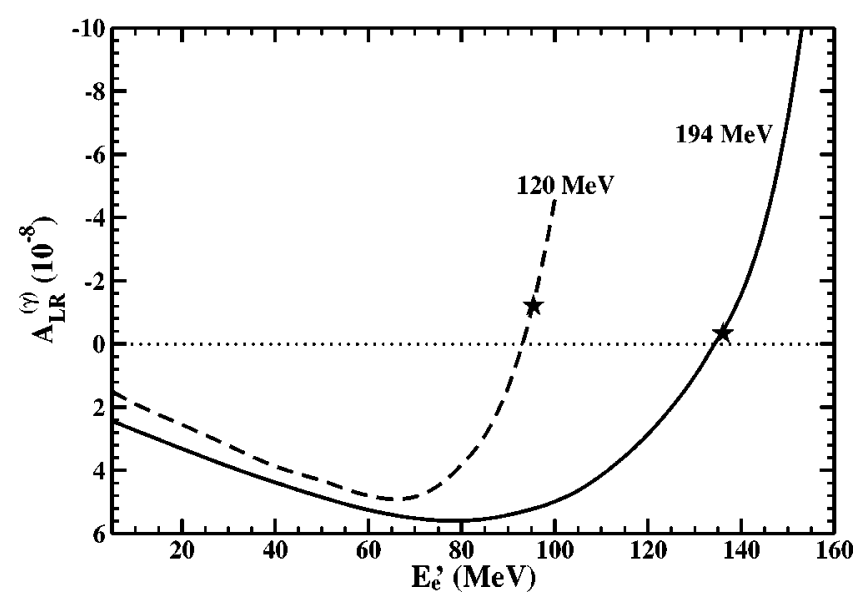

FIG. 9. The (a) total cross section and (b) two-body hadronic PV asymmetry versus electron final energy, for 194 and $120 \mathrm{MeV}$ incident beams and $180^{\circ}$ scattering angle. The asterisk denotes the position of the QE peak.

is determined by solving the Schrödinger equation, where $u(r)$ denotes the radial wave function, $k=\sqrt{m_{N} E}$, and the overall constant is fixed by the normalization condition $\left\langle E^{\prime} \cdots \mid E \cdots\right\rangle=\delta\left(E^{\prime}-E\right) \cdots$. This task is eventually reduced to integrating a one-dimensional differential equation for the radial component and solving for the phase shift. However, due to the tensor force, for $J>0$, states having quantum numbers $(L, S, J)=(J-1,1, J)$ and $(J+1,1, J)$ are coupled, requiring that one solve a coupled set of differential equations. The normalization of the radial wave functions is fixed by their asymptotic forms. For the uncoupled channel problem, one has

$$
u_{J L S}(r \rightarrow \infty)=r \sin \left(k r+L \pi / 2+\delta_{J L S}\right),
$$

where $\delta_{J L S}$ denotes the phase shift. For the coupled channel problem, the convention introduced by Blatt and Biedenharn (BB) [22], with two eigenphases shifts $\delta_{J}^{(1)}, \delta_{J}^{(2)}$ and a mixing parameter $\epsilon_{J}$, is adopted [29]. The two orthogonal, real solutions are 
(a)

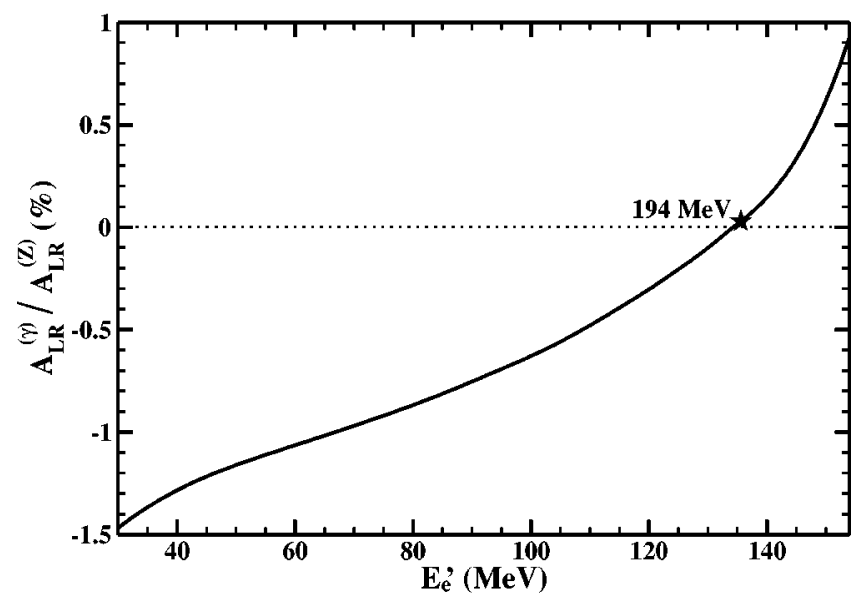

(b)

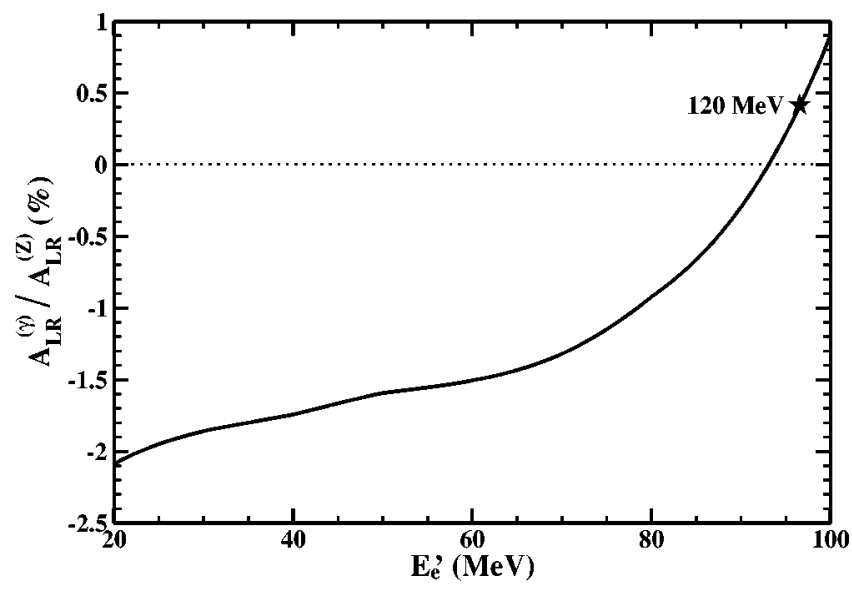

FIG. 10. The ratio of asymmetry due to two-body hadronic PV and $Z^{0}$ exchange, versus final electron energy: (a) $194 \mathrm{MeV}$ beam and (b) $120 \mathrm{MeV}$ beam. Both kinematics are the same as in Fig. 9.

$\left(\begin{array}{l}u_{L=J-1}^{(1)} \\ u_{L=J+1}^{(1)}\end{array}\right)(r \rightarrow \infty)=r\left(\begin{array}{c}\cos \epsilon_{J} \sin \left(k r+(J-1) \pi / 2+\delta_{J}^{(1)}\right) \\ \sin \epsilon_{J} \sin \left(k r+(J+1) \pi / 2+\delta_{J}^{(1)}\right)\end{array}\right)$,

$\left(\begin{array}{l}u_{L=J-1}^{(2)} \\ u_{L=J+1}^{(2)}\end{array}\right)(r \rightarrow \infty)=r\left(\begin{array}{c}-\sin \epsilon_{J} \sin \left(k r+(J-1) \pi / 2+\delta_{J}^{(2)}\right) \\ \cos \epsilon_{J} \sin \left(k r+(J+1) \pi / 2+\delta_{J}^{(2)}\right)\end{array}\right)$.

It should be noted that while we will still refer to solution $1(2)$ as ${ }^{3}[J-1]_{J}\left({ }^{3}[J+1]_{J}\right)$ state, it contains a component involving the other channel. We have verified our calculations by reproducing the experimental phase shifts.

The deuteron wave function,

$$
\left\langle\vec{r} \mid \mathcal{D}, M_{J}\right\rangle=\left\{\frac{u(r)}{r} \mathcal{Y}_{101}^{M_{J}}+\frac{w(r)}{r} \mathcal{Y}_{121}^{M_{J}}\right\} \chi_{0}^{0},
$$

is obtained by solving the eigenvalue problem for binding energy $E_{B}$ and $D / S$ ratio. The asymptotic and normalization conditions are

$$
\begin{gathered}
u(r \ll 1) \propto r, \quad u(r \gg 1) \propto r k_{0}(\gamma r) \\
w(r \ll 1) \propto r^{3}, \quad w(r \gg 1) \propto r k_{2}(\gamma r) \\
\int d r\left[u^{2}(r)+w^{2}(r)\right]=1 .
\end{gathered}
$$

Although one can follow a similar strategy and obtain the PV wave functions by the Green's function method mentioned in previous subsection, it is not straightforward to do so; the unperturbed wave functions are too complex to allow one to obtain analytical results as in the case of plane waves. Therefore, following the same approach as in Ref. [9], we directly solve the inhomogeneous equation, Eq. (33).

The basic idea is to solve the problem twice, once with the "source" term off (thus a homogeneous equation as solving the PC wave function) and then with the source term on. A general solution for the inhomogeneous equation, $\widetilde{\psi}_{g}$, can be expressed as a linear combination of solutions for the homogeneous equation, called the complimentary solutions, $\widetilde{\psi}_{c}(i)$, plus the particular solution, $\widetilde{\psi}$. Therefore, in order to obtain the particular solution,

$$
\widetilde{\psi}=\widetilde{\psi}_{g}-\sum_{i} \alpha_{i} \psi_{c}(i)
$$

the complimentary part has to be fully subtracted. Thus, we must determine $\alpha_{i}, i=1 \ldots N, N$ being the number of coupled equations.

In the case of solving scattering wave function, the asymptotic behaviors of both $\widetilde{\psi}_{g}$ and $\widetilde{\psi}_{c}$ can be expressed as linear combinations of incoming and outgoing spherical waves. While the interactions cause phase shifts of outgoing waves, the incoming waves are not altered. This observation tells us that, $\widetilde{\psi}$, the parity-mixed component induced by the PV $N N$ interaction, should not contain any incoming component. Using this result, $\alpha_{i}$ are the solution when the incoming wave components of $\widetilde{\psi}_{g}$ and $\widetilde{\psi}_{c}(i)$ completely cancel in Eq. (47).

Except for ${ }^{1} S_{0}$ and ${ }^{3} P_{0}$, which can only be mixed into each other, all the other uncoupled states, ${ }^{1,3} L_{J=L}$ could have mixtures from ${ }^{3}[L-1]_{L}$ and ${ }^{3}[L+1]_{L}$ states. For the coupled states, ${ }^{3}[L=J-1]_{J}$ and ${ }^{3}[L=J+1]_{J}$, both mix to ${ }^{1,3} J_{J}$. If the mixture is an uncoupled state, and we have

$$
\begin{aligned}
& \widetilde{\psi}_{c} \rightarrow a e^{-i(k r-L \pi)}+b e^{i(k r-L \pi)}, \\
& \widetilde{\psi}_{g} \rightarrow c e^{-i(k r-L \pi)}+d e^{i(k r-L \pi)},
\end{aligned}
$$

then

$$
\widetilde{\psi}=\widetilde{\psi}_{g}-\frac{c}{a} \widetilde{\psi}_{c}
$$

When the mixtures are coupled, a two channel calculation is needed. If one has 

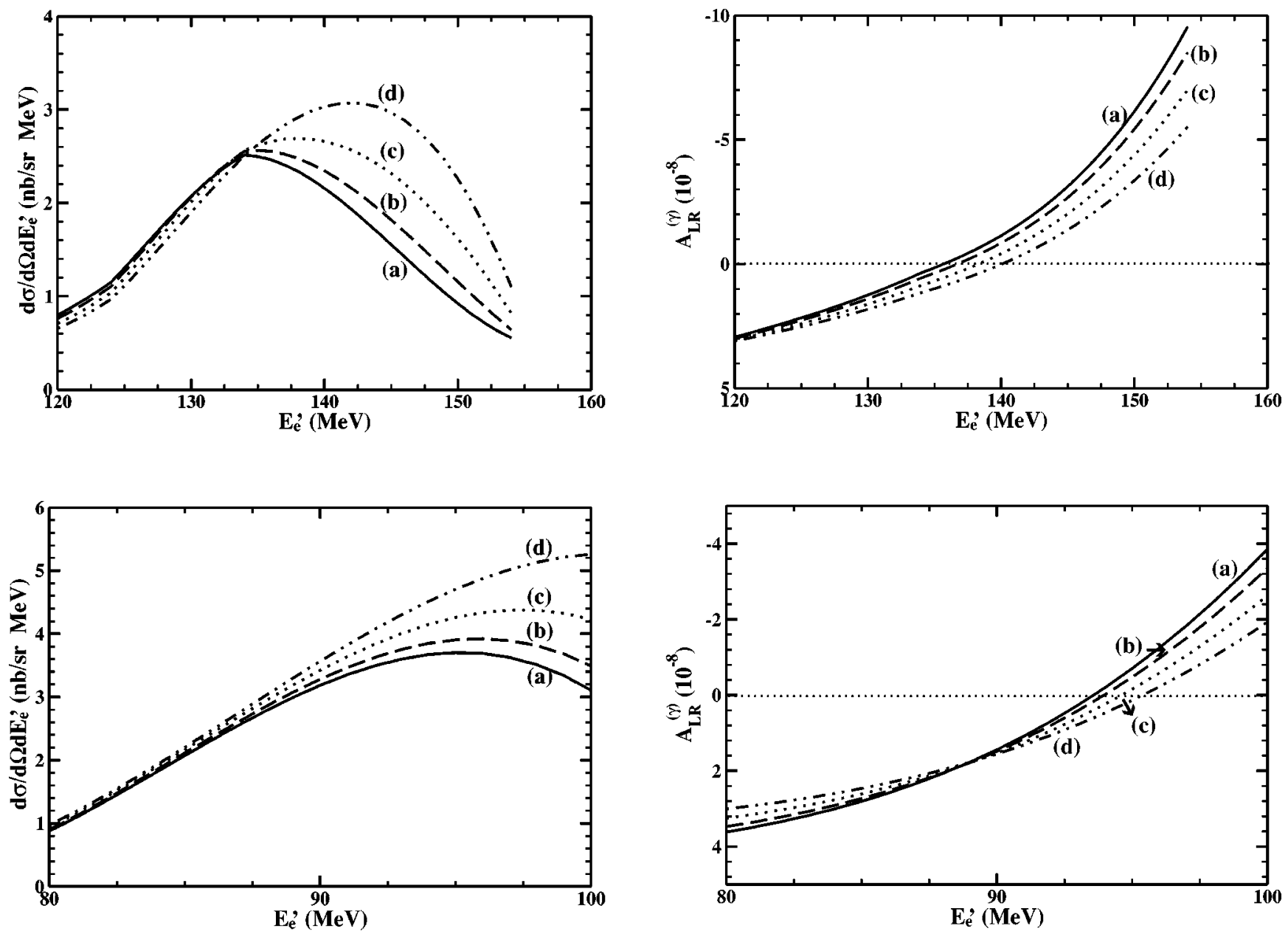

FIG. 11. The same plots as Fig. 9 with four average detector angles of SAMPLE experiments: (a) $160.5^{\circ},\left(\right.$ b) $154.0^{\circ}$, (c) $145.9^{\circ}$, and (d) 138.4 $4^{\circ}$ The left panels are for the $E_{e}=194 \mathrm{MeV}$ and right panels for the $E_{e}=120 \mathrm{MeV}$ case.

$$
\begin{aligned}
& \widetilde{\psi}_{c}(i=1,2) \rightarrow\left(\begin{array}{l}
a(i, 1) e^{-i(k r-(J-1) \pi)}+b(i, 1) e^{i(k r-(J-1) \pi)} \\
a(i, 2) e^{-i(k r-(J+1) \pi)}+b(i, 2) e^{i(k r-(J+1) \pi)}
\end{array}\right), \\
& \widetilde{\psi}_{g}(i=1,2) \rightarrow\left(\begin{array}{l}
c(i, 1) e^{-i(k r-(J-1) \pi)}+d(i, 1) e^{i(k r-(J-1) \pi)} \\
c(i, 2) e^{-i(k r-(J+1) \pi)}+d(i, 2) e^{i(k r-(J+1) \pi)}
\end{array}\right),
\end{aligned}
$$

then

$$
\begin{aligned}
\widetilde{\psi} & =\widetilde{\psi}_{g}(1)-\alpha_{1}(1) \widetilde{\psi}_{c}(1)-\alpha_{2}(1) \widetilde{\psi}_{c}(2) \\
& =\widetilde{\psi}_{g}(2)-\alpha_{1}(2) \widetilde{\psi}_{c}(1)-\alpha_{2}(2) \widetilde{\psi}_{c}(2)
\end{aligned}
$$

with

$$
\left(\begin{array}{l}
\alpha_{1}(i) \\
\alpha_{2}(i)
\end{array}\right)=\left(\begin{array}{ll}
a(1,1) & a(2,1) \\
a(1,2) & a(2,2)
\end{array}\right)^{-1}\left(\begin{array}{l}
c(i, 1) \\
c(i, 2)
\end{array}\right) .
$$

Note that all the coefficients here are complex. This implies that the mixed wave functions are also complex. However, in our framework, only the real part will contribute to the response function $W^{P V(\gamma)}$ and, thus, to the asymmetry.
Various criteria exist for testing the numerical solutions: (i) they should satisfy the differential equation, (ii) they should be independent of the initial conditions used to integrate the differential equation, (iii) they should be proportional to the source term, i.e., if the source term doubles, the solution should also double. These conditions are employed to make sure we obtain the correct solutions.

As for the parity admixture of deuteron, it is determined using the same procedure. Since one is dealing with a negative-energy state however, the asymptotic behavior is given by a linear combination of modified spherical Bessel functions, $i_{L}$ and $k_{L}$. The physically realistic solution is obtained by completely subtracting the $i_{L}$ component, because it diverges as $r$ increases.

\section{RESULTS AND DISCUSSION}

First, we compare the two approaches discussed in Sec. III. Figure 3 shows an example of the comparison between the plane wave scattering states and those obtained from the potential model calculations (in our case, it is $\mathrm{AV}_{18}$ ). Though at $E_{r e l}=1 \mathrm{MeV}$, the ${ }^{3} P_{0}$ solutions look almost the same, the plane wave ${ }^{1} S_{0}$ state differs from the more realistic solution 


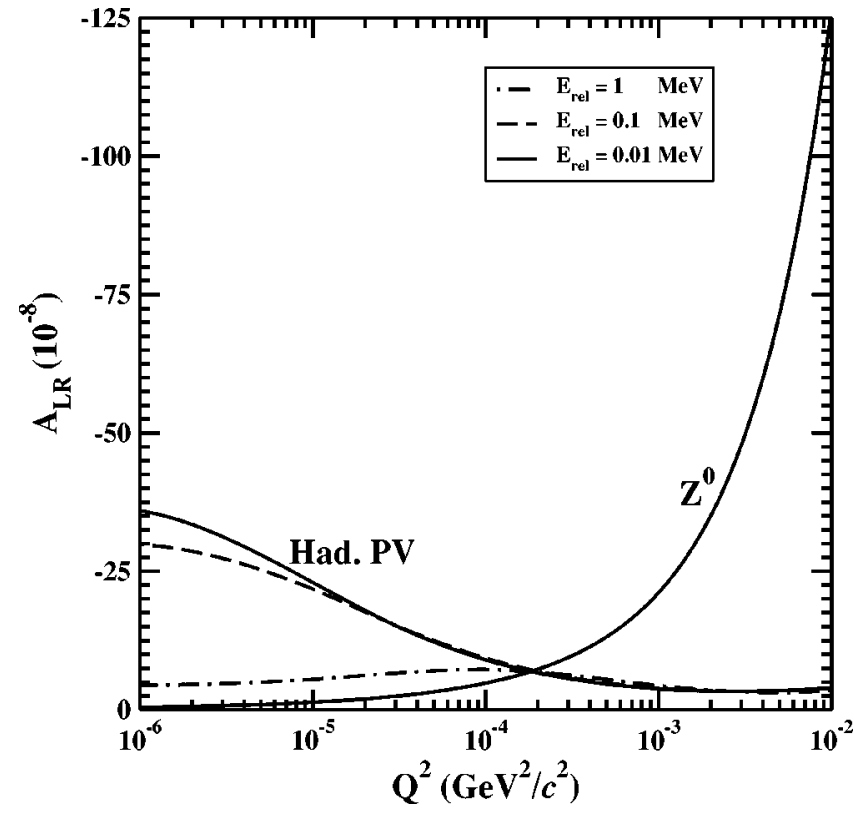

FIG. 12. The asymmetries due to hadronic PV and $Z^{0}$ exchange versus $Q^{2}$ in the threshold electrodisintegration region, with small, fixed $n p$ relative energies.

by a large phase shift as well as in its radial shape at small distances. Note that the latter difference is important because the PV $N N$ interaction is very sensitive to the short range behavior of wave functions. Therefore, the plane wave approximation is not adequate. Figure 4 shows the PV mixtures for these ${ }^{1} S_{0}$ and ${ }^{3} P_{0}$ states, ${ }^{3} P_{0}$ and ${ }^{1} S_{0}$, respectively. They are similar to the results of Ref. [9], which were obtained by using Reid soft-core potential, but differ slightly in magnitudes. For the deuteron mixing, the ${ }^{1} P_{1}$ state is induced only by $\rho$ and $\omega$ exchanges $(\Delta T=0)$, while the ${ }^{3} P_{1}$ is induced dominantly by the $\pi$ exchange; both results are plotted in Fig. 5. Also shown by the dotted lines in the same figure are the solutions of the plane wave Green's function. Though these curves are similar in shape, the potential model calculation gives smaller amplitudes and different small- $r$ radial dependence than plane waves. From now on, we only present results from the potential model calculation which are more realistic.

As the impact of $G_{A}^{(e)(T=1)}$ on $A_{L R}$ is more important at backward angles, we first examine the extreme case: $\theta$ $=180^{\circ}$. Subsequently, we present results relevant to SAMPLE kinematics. The maximum $Q^{2}$ we consider is 0.15 $(\mathrm{GeV} / \mathrm{c})^{2}$, and the saturation behavior shown in Fig. 6 justifies the truncation of the sum over final scattering states at total angular momenta $J_{f} \leqslant 7$.

Figure 7 indicates how the backward angle cross section and asymmetry vary with $Q^{2}$, ranging from 0.01 to $0.15(\mathrm{GeV} / c)^{2}$ at the QE peak. It is clear that the asymmetry due to two-body hadronic PV (plotted with a magnification of 100) is insignificant compared with the contribution of tree-level $Z^{0}$ exchange plus radiative corrections, which includes nucleon anapole effects. The curve for $Z^{0}$-exchange asymmetry, plotted using the static approximation result in

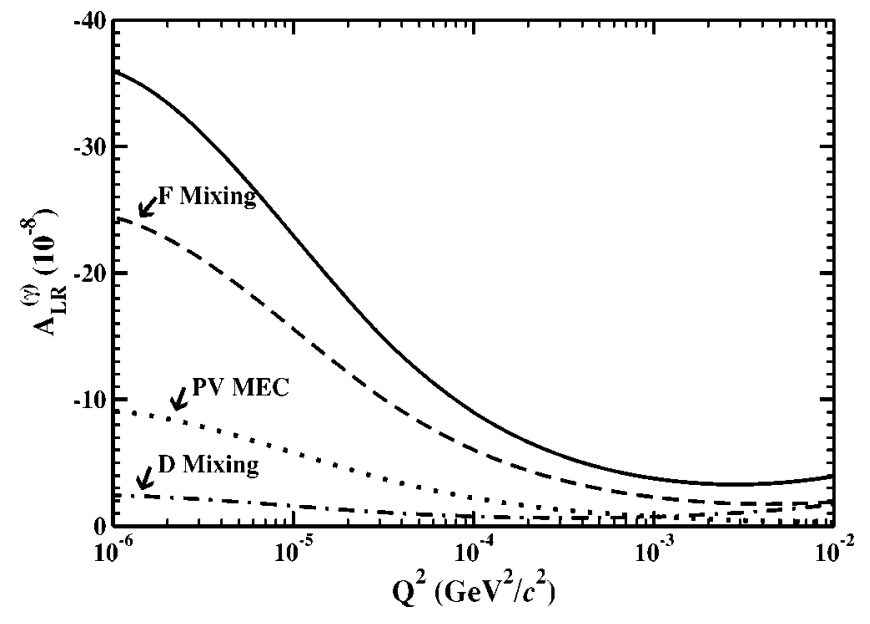

FIG. 13. The breakdown of various hadronic PV contributions to the asymmetry in the threshold electrodisintegration region, where $D, F$, and MEC refer to contributions from deuteron mixing, final state mixing, and PV meson exchange currents, respectively, and the solid line gives the total.

Ref. [1] with parametrized nucleon form factors, shows the expected proportionality to $Q^{2}$. The curve for hadronic PV shows a $0.05 \%$ correction to $A_{L R}^{(Z)}$ at $Q^{2}=0.1$ and a $0.3 \%$ correction at $Q^{2}=0.04$. We note that these results are consistent with simple scaling arguments as Eq. (30). Although there is some enhancement for $A_{L R}^{(\gamma)}$ as $Q^{2}$ decreases, even at $Q^{2} \sim 0.01$, near the threshold for $\mathrm{QE}$ kinematics, the correction is less than $5 \%$.

A detailed breakdown of various hadronic PV contributions is shown in Fig. 8. The deuteron mixing, rather insensitive to the $Q^{2}$ of the explored region, is the dominant contribution for $Q^{2} \geqslant 0.03$. Its correction to $A_{L R}^{(Z)}$ at $Q^{2}=0.1$ is $\approx 0.1 \%$, and $0.3 \%$ at $Q^{2}=0.04$. These values are consistent in the order of magnitude with the results of Ref. [10], which used Bonn potential to calculate the $\mathrm{PC}$ wave functions and the plane wave Green's function to calculate the parity mixture in deuteron. On the other hand, the final state mixing and PV meson exchange currents, though comparatively smaller, do have a combined contribution which could be as large as half of the contribution from deuteron mixing for $Q^{2} \geqslant 0.03$. They are also more sensitive to $Q^{2}$ and become important when approaching the QE threshold.

Away from the QE peak, the dependence of the cross section and asymmetry on final electron energy are shown in Fig. 9 for 194 and $120 \mathrm{MeV}$ beams. Since the scattered electrons are detected via the Cerenkov radiation (the threshold is about $20 \mathrm{MeV}$ ), scattered electrons with $E_{e}^{\prime} \lesssim 150$ and 100 $\mathrm{MeV}$, respectively, are detected. However, judging from the cross section plot, only regions about the peak energy \pm 20 and $10 \mathrm{MeV}$, respectively, are important for these two cases. When these asymmetries are further plotted as ratios to $A_{L R}^{(Z)}$, as shown in Fig. 10, we observe that the correction could become as large as a few percent. Notice, however, that the corrections change sign roughly when crossing the QE ridge. Hence, corrections from these two regions cancel after integration, thereby, keeping the total correction small. A similar 
feature was also found in the calculation of Ref. [23], where PC two-body effects were considered.

The setup of SAMPLE experiments actually cover the angular range from $130^{\circ}$ to $170^{\circ}$, the average angles of the detectors are: $138.4^{\circ}, 145.9^{\circ}, 154.0^{\circ}$, and $160.5^{\circ}$ [24]. The corresponding cross sections and asymmetries are plotted in Fig. 11. The general trend is that when the angle gets smaller, the cross section becomes larger and the asymmetry becomes smaller. However, the overall behaviors are not too different from the $\theta=180^{\circ}$ case.

Summarizing these observations, we conclude that the two-body hadronic PV effects in QE $e-d$ scattering are negligible. However, the situation changes in the kinematic region of threshold disintegration as shown in Fig. 12. At $Q^{2}$ $\sim 10^{-4}(\mathrm{GeV} / c)^{2}$, these two are comparable [30], and hadronic PV dominates when moving toward lower $Q^{2}$ region. Here again, the magnitude of $Q^{2}$ at which the hadronic PV and $Z^{0}$-exchange contributions are commensurate is roughly what one would expect based on the simple scaling arguments of Eq. (30). The detailed breakdown given in Fig. 13 shows that the final state mixing has the most important contribution and that PV meson exchange currents are also significant. The deuteron mixing, still rather independent of $Q^{2}$ evolution, becomes negligible. We also point out that while our calculation in this kinematic region is consistent with Hwang, Herley, and Millers [9] at $Q^{2}$ $\geqslant 0.0001 \mathrm{GeV}^{2} / c^{2}$, we obtain larger asymmetries as one approaches the threshold region. The reason is that we use a potential $\left(\mathrm{AV}_{18}\right)$ which has a much softer core than the Reid soft-core potential. Thus, the behavior of the wave function at low energy and small distance is important for studies of hadronic PV at threshold, including experiments like the photodisintegration of deuteron, radiative neutron capture, and neutron spin rotation.

\section{CONCLUSIONS}

The theoretical analysis of PV electron scattering asymmetries requires that one take into account effects that may, in principle, cloud the intended interpretation of an experimental result. In this study, we have analyzed the effects of parity violating $N N$ interactions that give rise to a nonvanishing inelastic $e-d$ asymmetry at the photon point. Our results indicate that for the QE kinematics relevant to the SAMPLE experiment, these effects generate a negligible contribution to the PV asymmetry. Moreover, contributions arising from each side of the QE peak produce cancellations when integrated over detector acceptances, thereby generating an additional suppression of the nuclear PV contamination. From this standpoint, then, the PV QE asymmetry provides a theoretically clean environment for studying electroweak nucleon form factors, such as $G_{A}^{(e)(T=1)}\left(Q^{2}\right)$. On the other hand, PV effects in the threshold region can become dominant, with asymmetries as large as a few $\times 0.1 \mathrm{ppm}$. Hence, near-threshold electrodisintegration or photodisintegration of the deuteron could provide a tool for probing the PV $N N$ interaction.

\section{ACKNOWLEDGMENTS}

We thank T. Ito, R. D. McKeown, T. W. Donnelly, and B. Jennings for useful discussions; J. Carlson, M. Paris, and R. Schiavilla for making available to us-prior to publicationthe results of their analogous computation of nuclear PV contributions to the QE asymmetry and for comments on our analysis; and the hospitality of Institute for Nuclear Theory where part of this work has been accomplished. C.-P.L. would also like to thank G. A. Miller for useful discussions on obtaining the wave functions. This work was supported in part under DOE Contract Nos. DE-FG03-02ER41215 and DE-FG03-00ER41132, and NSF Grant No. PHY-0071856.
[1] M.J. Musolf et al., Phys. Rep. 239, 1 (1994).

[2] R.D. McKeown and M.J. Ramsey-Musolf, hep-ph/0203011.

[3] B. Mueller et al., Phys. Rev. Lett. 78, 3824 (1997).

[4] D.T. Spayde et al., Phys. Rev. Lett. 84, 1106 (2000).

[5] R. Hasty et al., Science 290, 2117 (2000).

[6] S.L. Zhu et al., Phys. Rev. D 62, 033008 (2000).

[7] M.J. Musolf and B.R. Holstein, Phys. Lett. B 242, 461 (1990).

[8] W.-Y.P. Hwang and E.M. Henley, Ann. Phys. (N.Y.) 129, 47 (1980).

[9] W.-Y.P. Hwang, E.M. Henley, and G.A. Miller, Ann. Phys. (N.Y.) 137, 378 (1981).

[10] G. Küster and H. Arenhövel, Nucl. Phys. A626, 911 (1997).

[11] T. de Forest, Jr. and J.D. Walecka, Adv. Phys. 15, 1 (1966).

[12] J. D. Walecka, in Muon Physics, edited by V. W. Huges and C. S. Wu (Academic, New York, 1975), Vol. II.

[13] B. Desplanques, J.F. Donoghue, and B.R. Holstein, Ann. Phys. (N.Y.) 124, 449 (1980).

[14] E.G. Adelberger and W.C. Haxton, Annu. Rev. Nucl. Part. Sci. 35, 501 (1985).

[15] W. Haeberli and B. R. Holstein, in Symmetries and Fundamen- tal Interactions in Nuclei, edited by W. C. Haxton and E. M. Henley (World Scientific, Singapore, 1995), p. 17.

[16] W.C. Haxton, C.-P. Liu, and M.J. Ramsey-Musolf, Phys. Rev. Lett. 86, 5247 (2001).

[17] W.C. Haxton, C.-P. Liu, and M.J. Ramsey-Musolf, Phys. Rev. C 65, 045502 (2002).

[18] A.J.F. Siegert, Phys. Rev. 52, 787 (1937).

[19] J.L. Friar and S. Fallieros, Phys. Rev. C 29, 1645 (1984).

[20] M. J. Musolf, Ph.D. thesis, Princeton Univsersity, 1989.

[21] R.B. Wiringa, V.G.J. Stoks, and R. Schiavilla, Phys. Rev. C 51, 38 (1995).

[22] J.M. Blatt and L.C. Biedenharn, Phys. Rev. 86, 399 (1952).

[23] L. Diaconescu, R. Schiavilla, and U. van Kolck, Phys. Rev. C 63, 044007 (2000).

[24] T. Ito (private communication).

[25] H.P. Stapp, T.J. Ypsilantis, and N. Metropolis, Phys. Rev. 105, 302 (1957).

[26] Current conservation also implies the presence of PV electromagnetic two-body current contributions, as we discuss below.

[27] The extra $i$ for $\hat{T}^{\text {mag }}$ is introduced so that when real wave 
functions are used, the form factor $F_{M}$ is real.

[28] In Ref. [1], $\widetilde{F}_{X J}$ and $\widetilde{F}_{X J_{5}}$ are defined with an extra $1 / 2$ and $-1 / 2$ factor. It is found that the minus sign in Ref. [1] is a typographical error. The $1 / 2$ factor is absorbed here in the overall scale $A_{L R}^{0}$.

[29] The "nuclear bar" convention, defined in Ref. [25], is more commonly used in literature. However, the phase parameters of these two conventions are totally interchangeable. The reason for our choice is that all the wave functions are purely real in BB convention.

[30] The $Z^{0}$ asymmetry is plotted using the same formula from the static approximation. Although there are also two-body effects, the $Q^{2}$-dependence still governs the overall behavior. 